\title{
Verbesserung der Mundhygiene durch 3-Fach-Prophylaxe
}

Möchte man ein Anliegen des diesjährigen IDS-Auftritts von Listerine benennen, wäre es in jedem Fall „Verbesserung der Mundhygiene“. Das Unternehmen unterstütze nicht nur den Vortrag von PD Dr. Alexander Welk zum Thema „Effektivitätssteigerung des oralen Biofilmmanagements“, sondern stellte auch die neue Initiative 3-Fach-Prophylaxe auf der IDS vor. Es möchte die tägliche Mundpflege beste- hend aus Zahnbürste und Interdentalreinigung durch die zusätzliche Anwendung einer antibakteriellen Mundspülung langfristig verbessern. Dazu bündelt die Initiative Wissen zur täglichen Prophylaxe für eine optimale Mundhygiene, um dentalmedizinisches Fachpersonal und Zahnmediziner in ihrer alltäglichen Prophylaxe-Beratung in der Zahnarztpraxis zu unterstützen. Umfassende Informatio- nen und Serviceangebote stehen Dentalexperten auf der Online-Präsenz der Initiative www.listerineprofessional.de/initiative3-fach-prophylaxe zur Verfügung.

Nach einer Pressemit-teilung der Johnson \& Johnson GmbH, Neuss 